\title{
Zigbee Based Reconfigurable Clustered Home Area Network
}

\author{
Saad Ahmad Khan, Fahad Ahmad Khan, Arslan Shahid, Zubair Ahmad Khan \\ Al-Khawarizmi Institute of Computer Sciences, \\ Department Of Electrical Engineering, \\ University of Engineering \& Technology, Lahore Pakistan \\ saad.ahmad@uet.edu.pk, fahad.ahmad@uet.edu.pk, arslan.shahid@live.com, zubair.khan@kics.edu.pk
}

\begin{abstract}
Zigbee technology is here to stay. With current market trends it is to be understood that it will play a vital and major role in low rate wireless personal area networks. Zigbee networks facilitate numerous applications such as consumer electronics, home area networks, health care monitoring and environmental monitoring. Effective routing in wireless sensor networks is critical to conserve energy resources for the longevity of the network. In this paper we propose energy efficient cluster tree architecture for home area network. The reconfigurable architecture improves the Zigbee network performance by distributing equivalent load among its data fusing heads on basis of leftover energy. Simulations show that using such self organizing cluster tree topology is most effective in terms of energy consumption as compared to simple Zigbee mesh networks.
\end{abstract}

Index Terms - Zigbee; Clustering; Home Area Network; Home Automation; IEEE 802.15.4

\section{INTRODUCTION}

$\mathrm{P}_{\mathrm{t}}^{\mathrm{r}}$ rogress in the field of wireless communications has been tremendous during the last few decades. Using 802.11 standards for low cost solutions in wireless local area network (WLAN) and cellular networks have been provided and widely accepted. Low rate wireless personal area networks (LR-WPAN's) have come into existence for applications that require low cost, low power, low data rate using cost effective solutions. In recent time, the field of wireless personal area networking (WPAN), sensor networks in general and IEEE 802.15.4 in particular, has become the focus of extensive research. The applications related to this standard are focused to environment monitoring, home/office automation, industrial sensors and control, and other distributed sensor networks.

Various technologies have been introduced for implementation of wireless home networks. Some of the most widely used are IEEE 802.11, wireless LAN, Bluetooth, IEEE1394, UWB (ultrawide-band) based wireless solution and last but not least Zigbee IEEE 802.15.4. Earlier technologies were proprietary and not standardized among manufacturers. Zigbee is based on IEEE standard 802.15.4. Using Zigbee in home area networking gives access to handle a large network using a single coordinator, e.g., a single remote can give command to many devices and with ease to interface with outdoor mobile and remote devices. It is a more reliable, robust and cost effective solution. Zigbee is paving the way for intelligent sensors to provide greater control of lighting, heating, cooling, water and filtration, appliance-use and security systems from anywhere in and around the home. These controls dramatically improve energy efficiency with increasing power demands that exceed supply, as well as concerns around greenhouse gas emissions [1].

Using algorithms for finding efficient routing paths in home area networking takes in account many complications such as heavy and complex computations for the sustainment and management of good routing paths, which gives a perception of heavy energy consumption. Moreover, transmission cost of data is much and it is expedient to use clustering algorithms $[2][3][4]$. Keeping in view the properties desired for a home automation network, a clustered sensor network is more suitable. For example, using cluster head eliminates a large amount of redundant data in such a way that only aggregated data is sent to the sink [2].

In this paper we have introduced a Zigbee based reconfigurable cluster-tree network. We have done discussion on implementation of such networks in Zigbee, which is followed by our proposed Zigbee architecture for selforganizing cluster tree topology. We have shown through results that using Zigbee based clustered architecture gives the most optimum home area network in terms of power consumption. Our simulations clearly show the most optimum results in terms of reduced energy consumptions as compared to simple mesh and cluster tree Zigbee networks used for home area networking.

The rest of this paper is organized as follows: Section II is an overview of the related work in field of home area network Section III is a brief introduction to IEEE 802.15.4 keeping in view the beacon enabled aspects of Zigbee network. The Zigbee topology models are described in Section III, where we have explained our proposition for selecting a cluster tree model in home area networking. Section IV describes our home area network formation along with the distributed address assignment to keep unique address assignment scheme. Section V \& VI describe the proposed architecture, 
role of clustering and the software flow of our network. Our work in concluded in Section VIII.

\section{RELATED WORK}

In the domain of Wireless Sensor Networks, the first hierarchical clustering algorithm introduced was LEACH [15]. It utilized the random rotation of the cluster heads for even distribution of load among the sensor node. In PEGASIS [16], an extension and improvement in $\mathrm{LEACH}$, the chains were formed so that each node would be able to transmit and receive data from a neighbor and only one node is selected from that chain to transmit the data to the sink. HEED [17] considers the hybrid of energy and communication cost when selecting the cluster-heads. HEED considers only those sensors for cluster heads which have a high residual energy, unlike LEACH where cluster heads were randomly selected. An extension to basic HEED algorithm [18] was done by Huang and $\mathrm{Wu}$. In this modified version the re-execution of algorithm takes place for the orphaned nodes only. This significantly decrease the cluster-head count which in turn reduces the routing-tree-size needed during inter-cluster head communication and hence limits the data collection latency.

A Minimum Hop Routing Protocol [5] based on adaptive routing protocol was suggested by Chiang, Huang and Chang in which paths can be decided even if some nodes fail or energy is completely depleted from them. A routing table maintained in each node contains the parent, siblings, and child nodes. Flooding initiated the start of network, where a special packet helps in formation of table. The energy efficient selection of next-hop node is based on routing table.

GSM and Zigbee are incorporated [8] to establish a multimode network structure which employs GPRS as the data transmission device in the wide-area while Zigbee in the local area. Zigbee-GPRS "meter reading program" and "oil pipelining program" which are the basic ideas in it which are essential for commercial, industrial \& environmental applications but can be incorporated in home area networking after necessary modifications.

For a green house monitoring [9] system, Zigbee protocol had been introduced which is deployed using Jennic's chip JN5121. Using centric data algorithm technique a base station centric data gathering protocol BCDGP [10] was proposed. The basic entity for the proposed algorithm is WEN which is used for the flooding process and minimizes the total energy consumption is the network.

\section{IEEE 802.15.4 OVERVIEW}

The 802.15.4 is a part of the IEEE family of standards for physical and link-layers for wireless personal area networks. The WPAN working group focuses on short range wireless links, in contrast to local area and metropolitan area coverage explored in WLAN and WMAN working groups, respectively. The focal area of the IEEE 802.15.4 is that of low data rate WPANs, with low complexity and stringent power consumption requirements.
The first two layers of Zigbee protocol stack, i.e., physical (PHY) and media access (MAC) are defined by IEEE 802.15.4. Zigbee-compliant products operate in unlicensed bands worldwide, including $2.4 \mathrm{GHz}$ (global), $902-928 \mathrm{MHz}$ (Americas) and $868 \mathrm{MHz}$ (Europe). Device classification is used for complexity reduction. The standard differentiates between full function device (FFD), and reduced function device (RFD), intended for use in the simplest of devices. An RFD can only communicate with an FFD, whereas an FFD can communicate with both FFDs, and RFDs. There are four basic frame types defined in 802.15.4 and each data frame provides a payload of up to 104 bytes. The frame is numbered to ensure that all packets are tracked. A Frame-check sequence ensures that packets are received without error. This frame structure improves reliability in difficult conditions. Another important structure for 802.15.4 is the ACK frame. It provides feedback from the receiver to the sender, confirming that the packet was received without error. Finally, the beacon frame wakes up client devices, which listen for their address and go back to sleep if they do not receive it. Beacons are important for mesh and cluster-tree networks to keep all the nodes synchronized without requiring those nodes to consume precious battery power [11].

\section{Beacon Scheduling}

In multi-hop networks there's a possibility of collision of beacons frames or data transmissions of one device with its neighboring devices. To avoid this, beacon scheduling is necessary when implementation is done using cluster-tree topology. In Zigbee mesh networks, we don't need to take care of such issues as beaconing is not permitted in it. Our home area network is based upon beacon enabled transmissions so we need to take care of beacon scheduling.

\section{Scheduling method}

The home area network server (Zigbee coordinator) is responsible to set the beacon order and super-frame order of each device. One purpose of using beacon enabled mode in home area networking is to allow the nodes to sleep in order to conserve power, for doing so we need to set the beacon order much larger than the super-frame order. This helps to schedule active portion of super-frames of every device in neighborhood such that they are non-over lapping in time. This means that time is divided in approximately nonoverlapping time slots, and the active period of super-frame of every device in network occupies one of these nonoverlapping time slots [1]. A frame structure for a single beaconing device is shown in Figure 1.

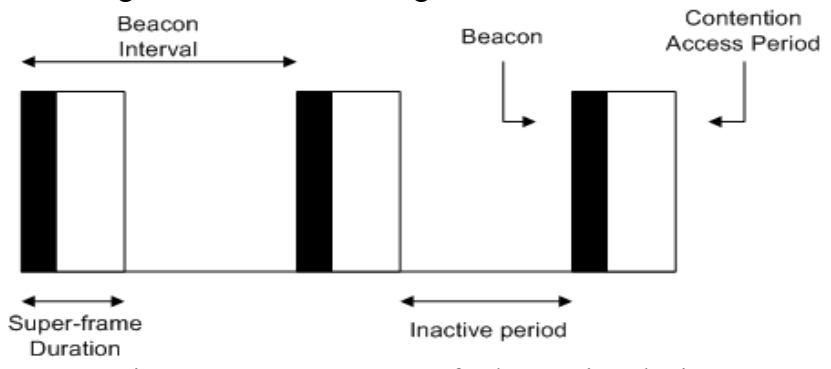

Figure 1- Frame structure for beaconing devices 
A device transmits its beacon frame at the start of the nonoverlapping time slot and transmit time is measured relative to the beacon transmit time of the parent device. Time offset is a part of beacon payload of every device in multi-hop network (home area network). When a device receives this beacon frame, it obtains both the beacon transmission time, i.e., of the neighboring device and parent of the neighboring device. The purpose of a device to obtain such information is to avoid the hidden node problem, i.e., a device will never transmit at the same time of transmission of parent of its neighbor.

\section{ZIGBEE TOPOLOGY MODELS}

Among several other components of Zigbee Network, the two main components are the FFD (Fully Functional Device) and the RFD (Reduced Function Device). At least there should be one fully functional device, i.e., the PAN coordinator. In our home area network model, the PAN coordinator is actually the server which is a computer and has FFD configured with it, which initializes and operates the whole of network.

The network models which are supported by Zigbee are Star topology, Peer-to-Peer topology and cluster tree topology.

\section{Star Topology}

Star topology is not adequate for home area network as the connection is established between the end-devices and one single controller only. For areas to be monitored at larger distance from the server it would require much energy consumption.

\section{Peer-to-peer (Mesh) Topology}

The advantage to using a peer-to-peer network is home area networking is that it allows transferring data using multi-hops to any other device in the network. In this type of network beacon mode is not supported, so we are not using mesh network for our home area network. Furthermore, we'll prove by results that using cluster-tree network is better option than to use mesh networks in home area network configuration.

\section{Cluster-Tree Topology}

In a cluster tree topology, there are multiple FFD and the RFD can connect to the network as leaf node to one of the FFD's. Synchronization services can also be provided to the end devices by any of the FFD which can also act as a router. In such a network, initially the PAN creates a network with a specific Cluster ID (CID) and other nodes maybe allowed to join at a later stage. After creation of network, the coordinator may instruct other FFD's to become cluster head of their adjacent nodes. We'll use such topology for home area networking, because it allows wide area network coverage, minimum energy consumption and also allows beacon mode transmissions.

\section{HOME AREA NeTWORK FORMATION}

\section{Initialization of network}

The home area network HAN-Server (Zigbee coordinator) initializes the network by sending the request to scan for any free or less occupied channels.

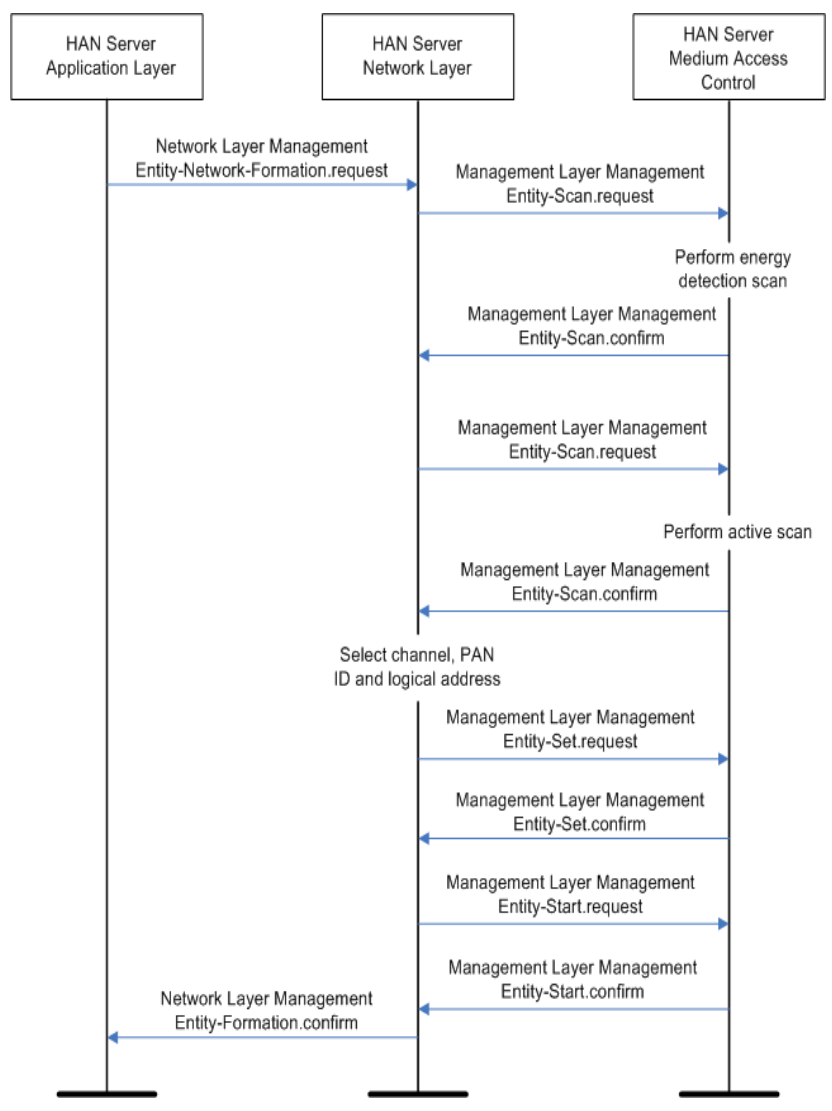

Figure 2- Formation of new network

The request is granted by the MAC layer and it scans a complete set of available channels and searches for possible interferers. After the results are communicated back, the network layer management entity orders the entire available channel in increasing order and discards those beyond the acceptable limit. Then a channel is selected which has the minimum number of interferers or favors that channel which has none other networks. After selection of channel a unique PAN identifier is chosen, after which network is established. Figure 2, explains the whole procedure of formation of a new network.

\section{Joining a network}

In our network, the parent-child relationship is created via the MAC layer association procedure which is initiated by the MLME-ASSOCIATE.indication primitive. When the procedure is initiated the NLME of parent determines, by searching through its neighbor table, for the existence of any entry of matching 64-bit address. If found so, it generates 16bit network address else it allocates the address from the allocated finite space address. The NLME informs the MAC layer of association which is replied back via the joining entity. The status of the response transmission to the child is communicated via MLME indication primitive. After successful transmission the NLME notifies the next higher layer by NLME-JOIN primitive. No reference to figure 3 . 


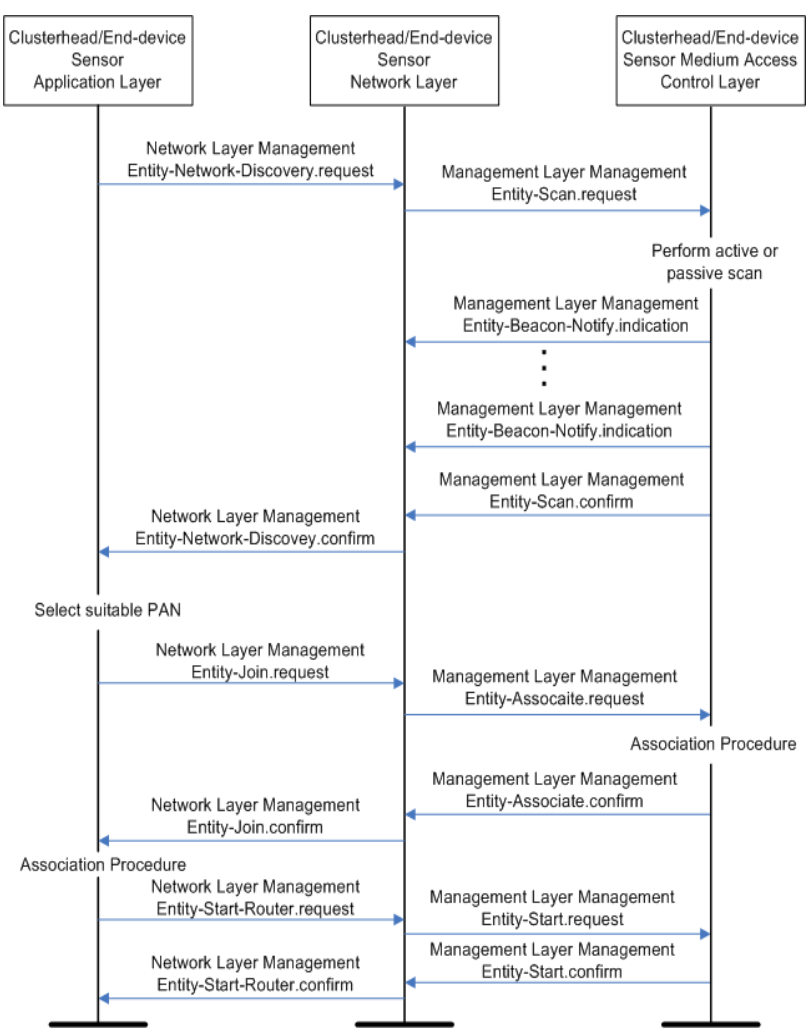

Figure 3 - Procedure of joining network through association

\section{Distributed Address Assignment Mechanism}

Zigbee has a distributed address assignment mechanism [14] which allots sub-blocks of finite addresses to parent node. The generated addresses that are assigned to the sub-children are unique within a particular network. The network identification base (NIB) manages the attributes that are required by a device. To configure distributed address mechanism, the default value of nwkUseTreeAddrAlloc is TURE. The Zigbee coordinator parameters determine the maximum number of children the network can have. A depth is associated with each device that indicates the number of hops for a transmitted frame to travel from parent to child. Zigbee coordinator has a depth 0 , and its children have depth 1 .

Home area network, is a multi-hop network, so maximum depth is greater than 1. The maximum depth is also determined by the Zigbee coordinator.

The Addr assign being distributed by each parent to its subchildren is given as:

$$
\begin{aligned}
& \mathrm{A}\left(\mathrm{d}_{\mathrm{nwk}}\right)= \\
& \left\{\begin{array}{c}
1+C_{\max } \cdot\left(d_{\max }-d_{n w k}-1\right) ; \text { if } R_{\max }=1 \\
\frac{1+C_{\max }-R_{\max }-C_{\max } \cdot R_{\max }\left(d_{\max }-d_{n w k}-1\right)}{1-R_{\max }} ; \text { otherwise }
\end{array}\right.
\end{aligned}
$$

Where,

$\mathrm{C}_{\max }=$ Maximum number of children in the network $\mathrm{d}_{\max }=$ Maximum depth of network

$$
\begin{aligned}
\mathrm{d}_{\text {nwk }}= & \text { Depth of network } \\
\mathrm{R}_{\max }= & \text { Maximum Number of routers a parent (cluster- } \\
& \text { head) may have as children }
\end{aligned}
$$

In home area networking, the end-devices such a temperature sensor, humidity sensor etc have an $A\left(d_{n w k}\right)$ value of 0 , because they are not capable of accepting any children. The cluster-heads having $A\left(d_{n w k}\right)$ value greater than 0 can accept more devices and assign addresses according to the routing and non-routing capability of the child-nodes. A cluster-head (parent) assigns an address that is one greater than its own, to its first router capable child device. Subsequently, $A\left(d_{n w k}\right)$ separates assigned addresses of router-capable devices.

The network addresses that are assigned to each end-device, (i.e., sensor) are in a sequential manner, i.e., for a $\mathrm{k}^{\text {th }}$ enddevice, address $A_{k}$ is given by;

$$
A_{k}=A_{\text {parent }}+A\left(d_{n w k}\right) \cdot R_{\max }+k
$$

where;

$$
1 \leq \mathrm{k} \leq\left(\mathrm{C}_{\max }-\mathrm{R}_{\max }\right) \text { and } \mathrm{A}_{\text {parent }}=\text { Address of parent }
$$

The $\operatorname{Addr}_{\text {assign }}\left(\mathrm{d}_{\mathrm{nwk}}\right)$ values for an example home area network are given in figure 4 .

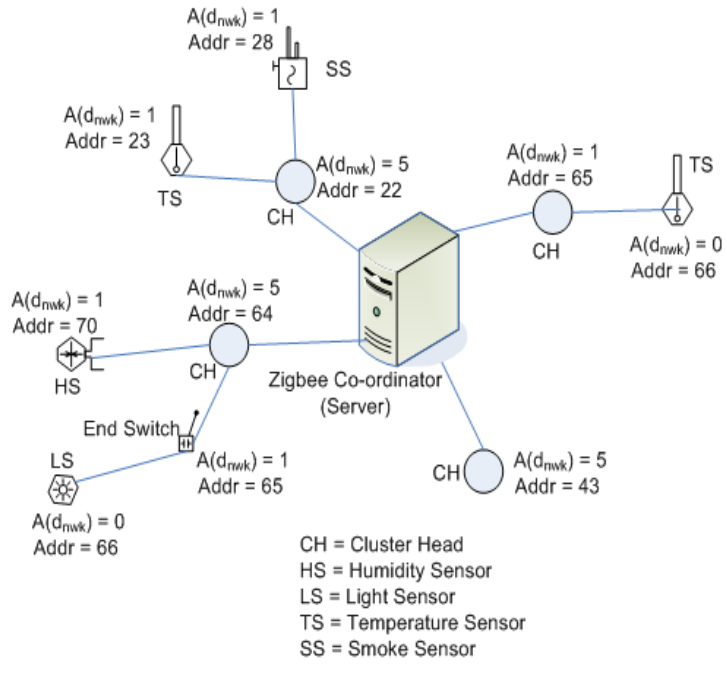

Figure 4 - Distributed Address Assignment Mechanism in Home Area Network

\begin{tabular}{|c|c|}
\hline Depth in the network, $\mathrm{d}_{\text {nwk }}$ & Offset Value, $\mathrm{A}\left(\mathrm{d}_{\mathrm{nwk}}\right)$ \\
\hline 0 & 21 \\
\hline 1 & 5 \\
\hline 2 & 1 \\
\hline 3 & 0 \\
\hline
\end{tabular}

Table 1- Offset address for each given depth in network

\section{RECONFIGURABLE CLUSTERED HOME AREA NETWORK}

Zigbee supports hierarchal modeling of a network. We have presented a cluster tree structure whose basic unit under each cluster head is star topology. It has been proved that star or simplified cluster tree topology best suits to low data rate and long battery life operations [12]. In our cluster based architecture, all nodes do not take part in the routing process. 
Data of ordinary nodes is gathered by the respective cluster heads. Cluster head has the exact knowledge of shortest routes to the coordinator. Our work is a dedicated effort on the network and application layer.

The main features of cluster based architecture for home area networking are [2]:

- Reduction is complexity of routing data to the base-station. Only the cluster-head need to have the information path of route to the base-station.

- The longevity of the network. As it is a load balanced algorithm, based on energy consumption calculated clusterhead, the life-time of nodes increases. The depletion of battery is taken into account and cluster-head is rotated among all members.

- The data can be efficiently aggregated and sent to basestation. It is due to this fact that usually a cluster-head would have similar node member in cluster vicinity. Similarity is on the base of sensing capabilities.

- The energy is conserved in such a network, because all nodes in a cluster send data to cluster-head, which is solely responsible for aggregating data and efficiently routing it to the base-station.

\section{Cluster head election phase}

After the establishment of network by the server (PAN coordinator) an election takes place in which every node participates. This election is for the selection of cluster heads of the network. A node ' $n$ ' would only be elected as a clusterhead if its energy density would be maximum in a neighborhood of defined radius 'r'. [13]

$$
\begin{aligned}
& \text { No. of clusters } \times \frac{\text { Energy of nodes }}{\text { Total energy of nodes in radius ' } r \text { ' }} \times \\
& \frac{\text { Radius ' } r \text { ' }}{\text { Total number of nodes }}
\end{aligned}
$$

\section{Node assignment phase}

After the formation of cluster heads, the remaining nodes join that cluster head which is at the least weighted distance as compared to other cluster heads. This phase of cluster formation is repeated until all the node is the network have established connection with the nearest cluster head.

\section{Minimum and Maximum Threshold Clause}

Due to the probabilistic approach for selection of cluster head, the formation of cluster might be uneven. To overcome such a situation, a minimum and maximum threshold is defined. If in a cluster the number of nodes is less than defined threshold then it would first split, and then the nodes would merge to a nearest cluster head. Similarly, if the number of nodes exceed from a particular threshold then it should split into smaller halves. Also a sensor is able to join another cluster head if it can get the shortest path to sink through the other cluster head.

\section{Balanced cluster head formation}

For a balanced clustered approach the nodes should rotate the cluster head formation between themselves. If a cluster-head has exhausted and can't further participate in network as a cluster-head then the cluster-head election within that cluster should again be head and new cluster head should be formed.

\section{Simulations}

For simulation purposes we had used the energy model of Zigbee based system on chip CC2431. The simulations performed on this network in on the area of $100 \times 100$ units and the base-station is a fixed one (having infinite energy), i.e., it is connected to the mains.

In Figure 6 and 7, the star indicates the base-station whereas the encircled dots represent the number of nodes alive after 200 rounds of transmissions and numbers of dots represent the number of nodes whose energy has been exhausted and cannot participate any further in the network.

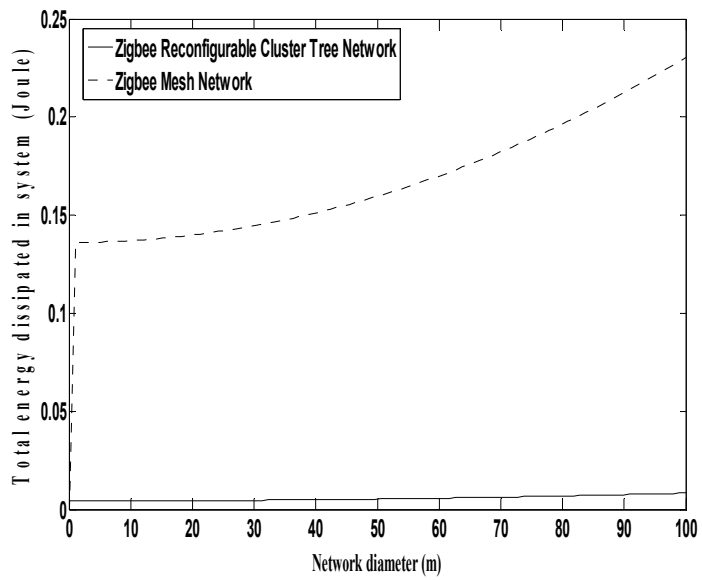

Figure 5 - Total energy dissipation verses increase in network diameter

To analyze our reconfigurable Zigbee cluster tree network, we use the following metrics:

\section{Total energy dissipated:}

Figure 5 shows the total energy dissipated within the network. It is shown that as the size of the network increases the cluster tree topology shows a stable behavior and its network energy consumption is considerably lesser than the simple Zigbee mesh topology.

\section{Number of nodes alive:}

Figure 6 and 7 show the total number of nodes that are left after a certain number of rounds of transmission. Initially we assumed a 100 node network. It is shown that after 200 rounds of transmission the number of nodes left in mesh network are far lesser as compared to the reconfigurable cluster tree network. Hence, the longevity of a network is increased using reconfigurable cluster tree network. 


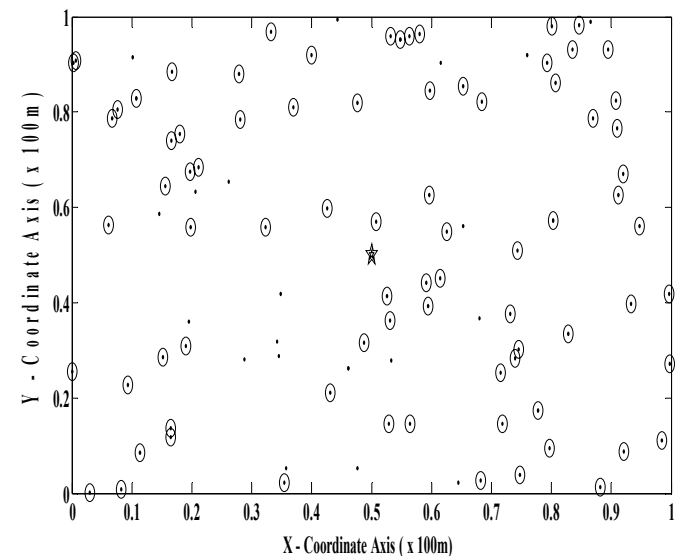

Figure 6 - Number of nodes alive after 200 rounds of transmission using reconfigurable cluster tree network

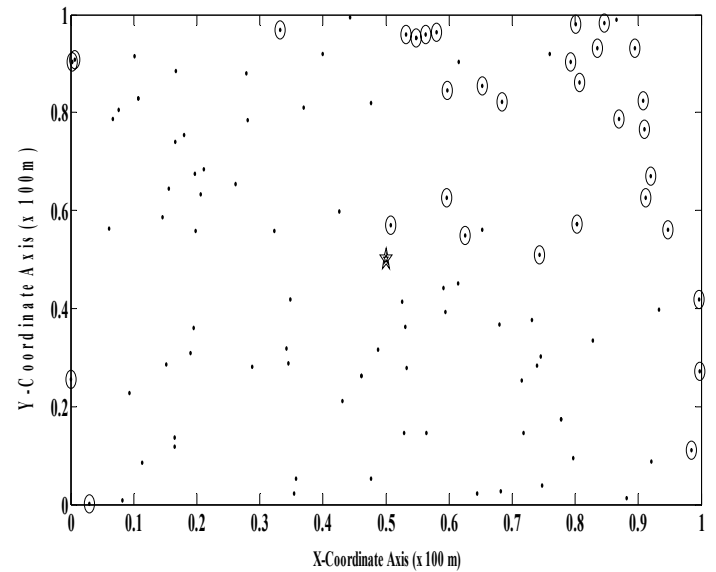

Figure 7 - Number of nodes alive after 200 rounds of transmission using star network topology

\section{CONCLUSION}

This paper describes a beacon enabled Zigbee cluster tree network that can reconfigure itself for minimum energy consumption in home area networks. We have established that a beacon-enabled hierarchically clustered topology is optimal in terms of power consumption and energy efficiency. Deployment of such a reconfigurable architecture outperforms mesh arrangements for a similar networking problem. We have concluded through simulation results that mesh networks waste a lot of energy as compared to our proposed cluster tree network. In our proposed schema, at different intervals, each node of the network volunteers to be a cluster-head based on its available energy factor. The idea of clustering revolves around data being aggregated at cluster head and then being fused to the base-station. Distributing energy in such a manner lends a hand in boosting the longevity of a network in terms of overall life-time. We believe that it is imperative for industry and academia alike, whom operate in the domain of WSNs and HANs, to understand the importance of clustering algorithms. An apt understanding and deployment of the approach will be beneficial and would result in magnitudes of power savor.

\section{REFERENCES}

[1] Zigbee market focus: Home control, www.Zigbee.org [March 30,2009]

[2] Kemal Akkaya and Mohamed Younis, "A survey on routing protocols for wireless sensor networks", Ad Hoc NetworksVolume 3, Issue 3, May 2005, Pages 325-349

[3] N. Narasimha Datta and K. Gopinath, "A survey of routing algorithms for wireless sensor networks", Journal of the Indian Institute of Science, 2006

[4] J. N. Al-Karaki and A. E. Kamal, "Routing Techniques in Wireless Sensor Networks: A Survey", IEEE Wireless Communications, $12 / 2004$

[5] Shao-Shan Chiang, Chih-Hung Huang, and Kuang-Chiung Chang, "A Minimum Hop Routing Protocol for Home Security Systems Using Wireless Sensor Networks," Consumer Electronics, IEEE Transactions on, vol.53, no.4, pp.1483-1489, Nov. 2007

[6] A. R. Al-Ali and M. AL-Rousan, "Java-Based Home Automation System", IEEE Transactions on Consumer Electronics, Vol. 50, No. 2, pp. 498-594, May 2004.

[7] Jae-Min Choi, Byeong-Kyu Ahn, You-Sung Cha, and Tae-Yong Kuc, "Remote-controlled Home Robot Server with Zigbee Sensor Network," SICE-ICASE, 2006. International Joint Conference, vol., no., pp.3739-3743, Oct. 2006

[8] Shen Lin, Shi xiangquan, and Ling Ming, "A wireless network based on the combination of Zigbee and GPRS," Networking, Sensing and Control, 2008. ICNSC 2008. IEEE International Conference on, vol., no., pp.267-270, 6-8 April 2008

[9] Zhou Yiming, Yang Xianglong, Guo Xishan, Zhou Mingang, and Wang Liren, "A Design of Greenhouse Monitoring \& Control System Based on Zigbee Wireless Sensor Network," Wireless Communications, Networking and Mobile Computing, 2007. WiCom 2007. International Conference on, vol., no., pp.2563-2567, 21-25 Sept. 2007

[10] Sung-Hwa Hong, Byongguk Kim, and Doo-Seop Eom, "A Basestation Centric Data Gathering Routing Protocol in Sensor Networks Useful in Home Automation Applications," Consumer Electronics, IEEE Transactions on, vol.53, no.3, pp.945-951, Aug. 2007

[11] Home networking with Zigbee by Mikhail Galeev http://www.embedded.com/columns/technicalinsights

[12] J. Zheng and M. J. Lee, "A Comprehensive performance study of IEEE 802.15.4,” IEEE Press Book, 2004.

[13] Lee SangHak, Ham KyungSun, and Park ChangWon, "Distributed Clustering for Wireless Sensor Networks," Communications and Information Technologies, 2006. ISCIT '06. International Symposium on, vol., no., pp.1113-1117, Oct. 18 2006Sept. 202006

[14] ZigBee Alliance. "ZigBee Specifications", version 1.0, April 2005.

[15] W.B. Heinzelman, A.P. Chandrakasan, and H. Balakrishnan, Application specific protocol architecture for wireless microsensor networks, IEEE Transactions on Wireless Networking (2002).

[16] S. Lindsey and C.S. Raghavendra, "PEGASIS: power efficient gathering in sensor information systems", in Proceedings of the IEEE Aerospace Conference, Big Sky, Montana, March 2002.

[17] O. Younis and S. Fahmy, "HEED: A Hybrid, Energy-Efficient, Distributed clustering approach for Ad Hoc sensor networks", IEEE Transactions on Mobile Computing 3 (4) (2004) 366-379.

[18] H. Huang and J. Wu, "A probabilistic clustering algorithm in wireless sensor networks", in Proceeding of IEEE 62nd Semiannual Vehicular Technology Conference (VTC), Dallas, TX September 2005 